\title{
Robert Koch, Aerztliche Erfahrungen in den Tropen.
}

Der Hauptzweck der Koch'schen Studienreise war die Erforschung der Malaria in Deutsch-Ostafrika. Der Malaria widmete denn auch Koch den grössten Theil seines bedeutungsvollen Vor. trages. Die Malaria, so führte er aus, ist die eigenste Krankheit der Tropen. Ungeheuer sind die Verheerungen, die sie alljährlich anrichtet. Wer nicht schon krank dort ankommt, der ist keinen Tag sicher, nicht von dieser Krankheit befallen zu werden. So sind vor einigen Jahren drei kräftige junge Leute nach Pangani gekommen, um dort eine Pflanzung anzulegen; sie wurden bereits nach drei Wochen schwer malariakrank in das I sazareth in Bagamoyo eingeliefert. In siechem Zustande kehrten sie später Ostafrika den Rücken. Sieben Trappisten, die nach dem Usambaragebiet entsandt worden waren, ist es nicht viel besser gegangen. Das Liebert'sche Vermessungscommando am Kilimandscharo verlor ebenfalls zwei Mann durch die Malaria, zwei andere mussten schwer krank zuräcktransportirt werden. Die Malaria begegnet uns in den Tropen auf Schritt und Tritt; sie befällt dell Soldaten auf dem Marsche ebenso wie den Beamten in seinem Bureau, der Ansiedler, der jalıraus, jahrein auf seiner einsamen Farm lebt, wird ebenso wenig von ihr verschont, wie der weite Strecken durchquerende Forschungsreisende. Nicht eher werden wir unserer Colonieen froh werden, als bis Mittel und Wege gefunden sind, um die Malaria wirksam zu bekämpfen. Um dieses Ziel zu erreichen, müssen wir uns zunächst über die Entstehung der Malaria volle Klarheit verschaffen.

Eine gewisse Aehnlichkeit mit der Malaria der Menschen besitzt eime Thierkrankheit, das Texasfieber der Rinder. Um die Malaria zu erforschen, hat Koch auch umfassende Studien übel das Texasfieber angestellt. Lange Zeit, so führt Koch aus, hat man über das eigentliche Wesen des Texasfiebers nichts erfahren können, bis Smith in dem Blut der befallenen Thiere einen Parasiten, das sogenannte Pirosoma bigeminum, von so charakteristischem Aussehen entdeckte, dass sich schon aus wenigen rothen Blutkörperchen die Diamnose stellen liess. Die Krankheit ist durch kleine Mengen Blut von einem Thier auf das andere übertragbar. Die eigentlichen Verbreiter der Krankheit sind aber, wie Smith nachgewiesen hat, eine Insectenart, die Zecken. Thiere aus dem Norden, welche mit Texasvieh zusammengebracht werden, erkranken nur dann, wenn Zecken von letzterem auf die ersteren hinübergelangen. Koch hat die Smith'sche Entdeckung in Ostafrika, wo das Texasfieber den Eingeborenen schon seit Jahrhunderten bekannt ist, nachgeprüft und bestätigt gefundell. Eine Lücke in der Smitl'schen Beweisführung ist aber von ihm durch folgenden Versuch ausgefüllt worden: Koch entnahm sowohl von gesunden als auch von krankell Thieren Zecken und schaffte diese unter strengster Trennung und Beobachtung aller Vorsichtsmaassregeln nach einem weit entfernt liegenden Ort, wo Texasfieber nicht vorkommt. Hier wurden nun gesunde Rinder mit den von den Zecken abgelegten Eiern geimpft, und siehe da, es erkrankten nach zweiundzwanzig Tagen von den geimpften Rindern nur diejenigen, welche mit kranken Zeckeneiern belegt worden waren. Die übrigen, mit gesunden Zeckeneiern geimpften Rinder blieben vom Texasfieber verschont. Thiere, welche frisch in Texas eingefiihrt wurden, reagirten nicht immer; sie sind deshalb aber nicht von vornherein als immun gegen die Krankheit anzusprechen. Die in Fiebergegenden aufgewachsenen Thiere machen das Fieber in der Regel in ihrer Jugrend, wennschon in leichterem Grade durch, wodurch sie wahrscheinlich immun werden. Diejenigon Thiere, 
welelle bei dem erwähnten Versuche das Fieber bereits einmal überstandell liatten, erkrankten zwar nach der Impfung mit kranken Zeckeneiern, jedocl war der Verlauf der Krankheit ein äusserst milder. Ist nun eine solche leichte Imınunität alısreichend gegen die scliwerere Form der Erkrankung? Koch ist der Meinung, dass, wenn diese Versuche weiter fortgefiihrt wïrden, manl zll cinem yünstigen Resultate gelangen würde. Die bisherigen $\mathrm{Er}$ fahrungen haben jedenfalls gelehrt, dass es möglich ist, eine kïnstliclle Immunität gegen Texasfieber zu erzeugen, und unan wird vielleicht zll einer Schutzlymple gegen das Texasfieber kommen.

In demselben Sinne wie das Texasfieber ist anch die Malaria als eine Blutparasitenkrankheit zu bezeichnen. Die Malaria ist in ihren verschiedenen Formen über die ganze Erde verbreitet. Der Krankheitsverlanf der Malaria ist in seiner charakteristisclıen Eigenart am bestell durch ständige Messungen der Körpertemperatur zll beobachten. Der Vortragende erläuterte dies graphiscli an der Hand typischer Fiebercurven. Dahei lob Koch die sehr interessante Thatsache hervor, dass die Quotidianaform aus zwei ineinandergeschobenen Tertianaformen besteht: bei der Blutuntersuchung vermag man zwei verschiedene Entwickelungsstadien des Malariaparasiten zll erkennen, von denell das eine dem lnmittelbaren, das andere dem rlächsttägigen Fieberanfall entspricht. Der tropischen Forn der Malaria ist anscheinend ein sehr nnregelmässiger Verlauf eigen. Nach den Beobachtungen der meisten Tropenärzte hat mall eine Norm für den Verlanf bislang nicht festzustellen vermocht. Dadurch unterscheidet sich die tropische, $d . h$. die Malaria in der scliwersten Form, anscheinend von der in Europa vorkommenden Kranklieitsart. Die Temperatırschwankungen wiederholen sich bei der Tropenmalaria tage-, ja wochenlang. Die Anfälle, welche vier. sechs, auch acht Stunden dauern, können sowohl einen um den anderen Tag, als auch täglich eintreten. Anch in Italien ist die Malaria während eines Theiles des Jahres, im Sommer, Spätsommer und Herbst in der scliweren Form bekaint; in den Tropen aber herrscht die schwere Form das ganze Jahr hindurcl. Ueber das Wesen der Malaria haben uns die Forschungen von Laveran und den italienisclien Aerzten Allfschluss gebracht. Man fand nämlich bei Malariakranken in den rothen Blutkörperchen einen eigenartigen Parasiten, der sich durch lebhafte Beweglichkeit auszeichnet und ziemlich rasch heranwächst. Der Höhepunkt seiner Entwicklıng fällt mit dem Höhepunkt des Fieberanfalls zusammen. Der Parasit nimmt allmählich ganz andere Formen an: er ist urspriinglich siegelringförmig, ballt sich zullächst zusammen, löst sich schliesslich jedoch in etwa 15-20 kleine Körperchen alf; letztere schwärınen wieder aus und nehmen von neuen Blutkörperchen Besitz. Es giebt in Dentsch-Ostaflika vier Arten seluwerer Tropenmalaria, zwei dieser Arten kommen, weil sie selır selten, wenig in Betracht. Von den anderen Formen kommt in DentschOstafrika die eine, die allch bei uns auftretende, leichtere Art der Malaria in etwa $10 \%$ aller Fälle, die andere, die schwerere Tropenform, in etwa 90\% aller Fälle vor. Aus der Form der allftretenden Parasiten kann man genaue Schlüsse anf das Stadium der Krankheit ziehen, man kann genau feststellen, ob die Krankheit schwer oder leicht ausfallen wird. In welcher näheren $\mathrm{Be}$ ziehıng die Parasiten zul den verscliedenen Krankheitsformen stehen, war bisher niclit ermittelt. - So ungefähr lagren die Verliältnisse, als $\mathrm{Koch}$ in Ostafrika anlangte.

Kocll machte das Studium des eigentlichen Tropenfiebers, also der schweren Form der Malaria, zu seiner besonderen Aufgabe. Er stellte zunächst über den eigentlichen Verlauf der Kranklieit Versuche an. Koch konnte nachweisen, dass auch dem tropischen Malariafieber eine ganz bestinmte Temperaturcurve z11kommt. sofern nämlicl der Krankheitsverlanf nicht durch Verabreichming von Chinin beeinflusst. wird. Bei dem etwa 36 Stunden wälırenden Anfall steigt zunächst die Temperatur rasch anl, hält sich nm etwa die Hälfte der Zeit auf gleicher Höhe, um dann etwas zil sinken, gleich darauf aber sofort allf die frïhere Höho anzısteigell. Auf dieser hält sie sich nun wieder die andere Hälfte der Zeit ınd sinkt dann erst wieder rasch zur normalen Grenze lierab. - Für die Behandlıng der tropischen Malaria hatte man bisher keinerlei sichere Anhaltspunkte; es blieb bislang weiter nichts übrig, als ganz blindlings Chinin zll geben. Der Tropenarzt giebt den Kranken, sofern er eine andere Krankheit nicht nachweisen kann, Clinin anf den ganzen Tag vertheilt. Dieses Tasten wird nun allfhören können, denn wir wissen jetzt genall, waun wir den Parasiten am sichersten treffen können, in welehem Moment das Chinin anzuwenden ist. Es ist das der Sporulation voraufgehende Stadium der Plasinodienentwickelung. Das Chinin iibt anf die Parasiten keine tödtliche Einwirkıng ans, wohl abel wirkt cs leemmend auf ihr Waclisthum ein. Da sicl die Zeit des Anfalles aber nach dem Befunde der mikroskopisclien Blintıntersuchung mit voller Sicherheit feststellen lässt, so ist damit der Termin der Chininverabreichung fixirt. Chinin zur lechten Zeit gegebell, leilt die tropische Malaria. Und weil wir eben jetzt wissen, dass das Tropenfieber verhältnissmässig leicht zll heilen ist, deswegen hat es aucl seine Schrecken fïr lins zum allergrössten Theil verloren. Ko ch hat die Richtigkeit dieser Beobachtung in allen Fällell, in welchen ihm Malariakranke zur Behandlung zugefïhrt wurden, bestätigt gefınden. Nur zwei, ihm allerdings erst in vollständig hoffnungslosem Zustande übergebene Kranke konnten nicht mehr geheilt werden. Diese Erfallrung hat aucl Einfluss anf die Sanatorienfrage. Man hat vorgesclilagen, da Malariakranke nach 4-6 Wochen oft sehwere Riickfälle zu erleiden haben, Sanatorien im Gebirge zu errichten. Derartigen Sanatorien wird nach Koch's Meinung ein zll grosser Werth beigelegt. Es ist nach Koch ganz gleichgültig, ob derjenige, welcher Parasiten in seinem Blute hat, sich in der Ebene, an der Küste, oder im Gebirge befindet. Solange das Blut nicht parasitenfrei ist, bekommt er eben stets wieder Fieber, ganz llnbbhängig von der Gegend, in welcher er sich aufhält. Kranken, welche das Fieber einınal überstanden haben, giebt man zurr Verhïitıng von Rückfällen sowie als Vorbengungsmittel Chinindosen von $1 \mathrm{~g}$ in Zwischenrälımen von je fünf Tagen. Es genügt dies vollkommen, um einen Ausbruch des Fiebers zll verhindern. Kürzer als fïnf Tage dïrfen die Pausen nicht sein, anch muss das Chinin etwa $1^{1 /}, 2$ - Monate lang genominen werden. Aber auch hier sind umfangreiche Versuche nocl erforderlich. Die endgültige Festsetzung derartiger Maassregeln kann bei zielbewlisster Inangriffnahne der Forschung nur eine Frage der Zeit sein. Eigenthïmlich ist der Umstand, wie schnell sicl ein Kranker nach Ueberstelien allch des schwersten Fiebers erholt und seinen Gescliäften wieder nachgelien kann.

Ulkklar war bisher die Beziehung des sogenannten Scliwarzwasserfiebers zur Malaria. Man hat sich bislang nicht von dem Gedanken trenıen können, dass bei dem Schwarzwasserfieber die Malaria eine Hauptrolle spielt. Der Vortragende kommt auf Grund seiner Bcobaclitungen zll dem Schlusse, dass Schwarzwasserfieber und Malaria niclits miteinander zu thun haben. In typischen Fällen von Schwarzwasserfieber sind niemals Malariaparasiten nachgewiesen worden. Viel eher ist anzunehmen, dass das Schwarzwasserfieber mit der Chininbehandlung zllsammenhängt. Mindestens müsse man sagen, dass kein Fall von Schwarzwasserfieber beobachtet ist. in dem mit Sicherheit eine Chininvergiftung alsgeschlossen werdell konnte.

Der Grundsatz unserer Hygieniker: Kranklieiten vorbeugen, ist besser als Krankheiten heilen, kann anch auf die Malaria berechtigte Anwendung finden. Bezüglich der Entstehnng und U ebertragung dieser Krankheit haben wir wohl Vermuthungen, aber wir sehen noch nicht klar nach dieser Richtung. Wir mïssen erst wissen, wie die Parasiten in den Menschen liineinkommen. Geschieht die Uebertragung etwa durch das Wasser, durch die Luft? Eine direkte Uebertragung von Mensch zu Mensch ist nicht möglich, denn sonst müsste die Malaria ansteckend sein. Die Malariainfection muss also anf einigen Umwegen stattfinden. Eine Uebertragung der $\mathrm{Pa}$ rasiten durch die Luft ist undenkbar: die schnelle Eintrocknıng des Blutes und der in ihm enthaltenen Parasiten macht einen derartigen Verbreitungsmodus unmöglich. Dass die Infection des Menschen nicht durch das Wasser geschielt, ist ebenfalls direkt bewiesen worden. Man hat Sumpfwasser in Malariagegenden trinken lassen, ohne dass eine Infection erfolgt ist. Allffallen müssen dagegen die Beziehıngen blıtsangender Insecten, der Mosquitos, zur Verschleppung der Malaria. Die Malariainfection geschielit hauptsächlicl in der Nachtzeit. Wo Mosquitos fehlen, tritt niemals Malaria auf. $S_{0}$ ist z. B. eine kleine Insel im deutsch-ostafrikanischen Besitze mosquitofrei und auch malariafrei. Ebenso ist das Usanbaragebiet von einer bestimmten Höhe ab malariafrei: es giebt auch dort keine Mosquitos. Wir haben in letzter Zeit zahlreiche Krankheiten kennen gelernt, bei welchen die Uebertragung durch blutsaugende Insecten geschieht. Für das Texasfieber ist der sichere Beweis geliefert. Deshalb hat anch diese Uebertragungstleorie bei der Malaria so viel Wahrscheinlichkeit für sich. Wir dïrfen uns aber die Sache nicht so vorstellen, dass der Mosquito zuerst den kranken und dann den gesunden Menschen sticht und letzteren dadurch inficirt. Die Infection muss vielnehr auf indirektem Wege geschelıen. Die Uebertragung geschieht walırscheinlich so, dass die Mosquitos z11nächst ihre Eier verschleppen. In diesen anf Menschen verpflanzten Eiern entwickeln sicl sodann die Malariaerreger. Die Kranklıeit kommt auch nicht direkt zum Alsbruch, also etwa am anderen Tage, nachdem der Mosquito den Menschen gestochen hat. Z11 einem Fieberanfall gehören vielmehr eine ganze Anzahl von Parasiten; sie mïssen sich also erst im Blute des Menschen vermehren. Es verstreicht immer erst eine längere Zeit von der Infection durch das Insect bis zum Ausbruch der Krankheit, nnd zwar 
kann man diese Frist auf etwa 10-14 Tage annehmen. Das muss sehr bei der Behandlung berücksichtigt werden.

$\mathrm{Ob}$ es eine Immunität gegen Malaria giebt? Diese bislang nicht entschiedene Streitfrage glaubt Kocli bejahen zu müssen. Es giebt keinen Menschen, der von Anfang an immun ist. Malariakranke, welche, ohne mit Chinin behandelt zu sein, das Fieber überstanden haben, besitzen späterhin eine gewisse Immuniät. Die Immunität kann aber auch auf natürlichem Wege erworben sein. Das beste Beispiel hierfür bilden die Neger. Der Bergneger weiss recht wohl, dass, wenn er seine Berge verlässt und sich an die Küste begiebt, er von der Malaria befallen wird, und $z$ war in der Regel ausserordentlich schwer. Kommt er jedoch durch, so liat er damit die Immunität erworben und kann von da $a b$, ohne befürchten zu müssen, sich aufs neue zu inficiren, das Küstengebiet betreten. Der Küstenneger bekommt deshalb nicht das Fieber, weil wahrscheinlich schon seine Voreltern gegen die Krankheit immun waren. Ganz ähnliche Verhältnisse bestehen auch bei den Indern. Diese bekommen die Malaria ebenfalls ausserordentlich schwer. Man findet aber auch Tausende von Indern, die anscheinend gegen Malaria immun sind. Es unterliegt daher auch keinem Zweifel, dass eine besondere Immunität vorhanden ist. Bei anderen Krankheiten, wie bei der Rinderpest, der Hundswuth, ist es uns gelungen, künstliche Inımunität herzustellen, weshalb sollte dasselbe bei der Malaria nicht der Fall sein? Giebt es eine natürliche Immunität gegen Malaria, so steht zu erwarten, dass es in abselıbarer Zeit auch ein Verfahren zur künstlichen Immunisirung geben wird.

Die bisher gebräuchlichen, Maassregeln zur Bekämpfung der Malaria, z. B. die Trockenlegung von Sümpfen, die Anpflanzung von Eucalyptus, beseitigen die Gefahr keineswegs. Die augenblicklich wichtigste Maassnahme für unsere Colonieen ist die Entsendung einer genügenden Anzalll gut geschulter Aerzte, um planmässig den Chiningebrauch zur Durchführung zu bringen. Die Anwendung des Chinins ist keineswegs so einfach, wie man es sich vorstellt, deshalb kann mall auch Nichtärzten das Chinin zum beliebigen Gebrauch nicht ohue weiteres in die Hände geben. -.. Aber auch in mancher anderen Beziehung lässt sich recht viel thun. So ist die Schaffung gesunder Wohnräume, in welche die Mosquitos nicht hineiudringen können, von grosser Wichtigkeit. Wenn es uns gelingt, der Malaria Herr zu werden, so ist dies gleichbedeutend mit der friedlichen Eroberung eines der schönsten und fruchtbarsten Theile unserer Erde für unser Vaterland. Wir schicken Expeditionen nach dem Nordpol und nach dem Südpol. Wichtiger muss uns aber doch die Erschliessung unserer Colonieen erseheinen und das geschieht am ehesten durch eine planmässige und zielbewusste Malariaforschung. $\quad$ P. Herzfeld (Berlin). 